Denkstrukturen in Lögsungsansätzen von Modellierungsaufgaben 

Xenia-Rosemarie Reit

\section{Denkstrukturen in \\ Lösungsansätzen von \\ Modellierungsaufgaben}

Eine kognitionspsychologische Analyse schwierigkeitsgenerierender Aspekte

Springer Spektrum 
Xenia-Rosemarie Reit

Frankfurt, Deutschland

Dissertation zur Erlangung des Doktorgrades der Naturwissenschaften angenommen vom Fachbereich 12 der Johann Wolfgang Goethe-Universität in Frankfurt am Main, 2015

D30

Dekan: Prof. Dr. Uwe Brinkschulte

Erstgutachter: Prof. Dr. Reinhard Oldenburg

Zweitgutachter: Prof. Dr. Gilbert Greefrath

Datum der Disputation: 10.12.2015

ISBN 978-3-658-13188-3

ISBN 978-3-658-13189-0 (eBook)

DOI 10.1007/978-3-658-13189-0

Die Deutsche Nationalbibliothek verzeichnet diese Publikation in der Deutschen Nationalbibliografie; detaillierte bibliografische Daten sind im Internet über http://dnb.d-nb.de abrufbar.

Springer Spektrum

(C) Springer Fachmedien Wiesbaden 2016

Das Werk einschließlich aller seiner Teile ist urheberrechtlich geschützt. Jede Verwertung, die nicht ausdrücklich vom Urheberrechtsgesetz zugelassen ist, bedarf der vorherigen Zustimmung des Verlags. Das gilt insbesondere für Vervielfältigungen, Bearbeitungen, Übersetzungen, Mikroverfilmungen und die Einspeicherung und Verarbeitung in elektronischen Systemen.

Die Wiedergabe von Gebrauchsnamen, Handelsnamen, Warenbezeichnungen usw. in diesem Werk berechtigt auch ohne besondere Kennzeichnung nicht zu der Annahme, dass solche Namen im Sinne der Warenzeichen- und Markenschutz-Gesetzgebung als frei zu betrachten wären und daher von jedermann benutzt werden dürften.

Der Verlag, die Autoren und die Herausgeber gehen davon aus, dass die Angaben und Informationen in diesem Werk zum Zeitpunkt der Veröffentlichung vollständig und korrekt sind. Weder der Verlag noch die Autoren oder die Herausgeber übernehmen, ausdrücklich oder implizit, Gewähr für den Inhalt des Werkes, etwaige Fehler oder Äußerungen.

Gedruckt auf säurefreiem und chlorfrei gebleichtem Papier

Springer Spektrum ist Teil von Springer Nature

Die eingetragene Gesellschaft ist Springer Fachmedien Wiesbaden GmbH 


\section{Danksagung}

Mein Dank gilt Prof. Dr. Reinhard Oldenburg für die Unterstützung und das Vertrauen während der Anfertigung dieser Arbeit. Weiterhin möchte ich mich bei Prof. Dr. Gilbert Greefrath, für sein entgegengebrachtes Interesse an meiner Arbeit bedanken.

Durch die finanzielle Unterstützung der Stiftung Polytechnische Gesellschaft war es mir möglich, all meine Zeit der Anfertigung dieser Arbeit zu widmen. Ich bin nicht zuletzt wegen der Seminare und der Möglichkeit des wissenschaftlichen Austauschs mit anderen, sehr dankbar Teil der polytechnischen Familie zu sein.

Weiterhin gilt mein Dank allen Kollegen, die meine Arbeit vorangebracht haben. Hanna, vielen Dank für die dringend nötigen Kaffeepausen und deine unermüdliche Motivation dich in meine Themen hineinzudenken.

Ganz herzlich möchte ich mich bei Matthias Ludwig für seine unendliche Geduld, die konstruktiven Antworten auf all meine Fragen und den Rückhalt während der letzten drei Jahre bedanken.

Meine Familie und Freunde standen mir mit Rat und Tat beiseite. Mein besonderer Dank gilt meinem Vater Hans-Gerd Reit für die fordernden Fragen, kritischen Diskussionen und die geduldige Unterstützung all meiner Pläne.

Abschließend möchte ich mich bei den fleißigen Korrekturlesern Jana, Henrieke, Verena und Lukas bedanken. 



\section{Zusammenfassung}

Unter mathematischem Modellieren wird, vereinfacht ausgedrückt, der Prozess des Bearbeitens einer realen Problemsituation, unter Verwendung von Mathematik, verstanden. Als eine von sechs mathematischen Kompetenzen, wird dem mathematischen Modellieren eine bedeutende Stellung in den Bildungsstandards zuerkannt. Auch auf internationaler Ebene ist mathematisches Modellieren in Vergleichsstudien wie z.B. PISA (Programme for International Student Assessment) etabliert. Trotz des bildungspolitischen und fachdidaktischen Konsens über die Wichtigkeit mathematischen Modellierens in der Schule, zeigen einige Studien, dass der Anteil mathematischen Modellierens am Alltagsunterricht gering ist. In diesem Zusammenhang geht aus mehreren Untersuchungen hervor, dass dem mathematischen Modellieren im schulischen Kontext wenig Bedeutung beigemessen wird, wohingegen technische Aufgaben im Mittelpunkt des Unterrichtsgeschehens stehen. Eine detaillierte Auseinandersetzung mit den Implikationen einer unterrichtlichen Integration mathematischen Modellierens, bringt Schwierigkeiten und Hindernisse zu Tage, welche in Zusammenhang mit speziellen Charakteristika von Modellierungsaufgaben zu sehen sind. Eine Schwierigkeit ist die Vielfalt der zur Lösung möglichen Lösungswege und die damit verbundene Anwendbarkeit verschiedenster fachmathematischer Kenntnisse. Das führt dazu, dass Modellierungsaufgaben in vielerlei Hinsicht zunächst wenig überschaubar sind.

Die Ungleichheit zwischen bildungspolitischen Vorgaben und Schulalltag zeigt, dass vor allem diesbezüglich Handlungsbedarf besteht. Die vorliegende Arbeit knüpft an diese Problematik an, indem auf Basis tatsächlicher Schülerlösungen ${ }^{1}$, eine systematische Erarbeitung schwierigkeitsgenerierender Aspekte in Lösungsansätzen von Modellierungsaufgaben, insbesondere aus kognitionspsychologi-

\footnotetext{
${ }^{1}$ In der Arbeit wird der generische Maskulin verwendet. Dieser schließt aber stets auch die weibliche Form mit ein.
} 
scher Sicht, erfolgt. Schülerlösungen, welche z.B. auf dem gleichen mathematischen Modell beruhen, werden zunächst zu Lösungsansätzen zusammengefasst. Anschließend wird die kognitive Struktur von Lösungsansätzen auf parallele bzw. sequentielle Denkoperationen hin untersucht. Eine, aus der Kognitionspsychologie abgeleitete Hypothese ist, dass ein Zusammenhang zwischen der Anzahl parallel durchzuführender Denkoperationen und der Schwierigkeit des jeweiligen Lösungsansatzes besteht. Ziel ist es, darauf aufbauend eine tragfähige Methode zu entwickeln, welche eine valide theoretische Einschätzung der Schwierigkeit von Lösungsansätzen erlaubt. Zusätzlich wird ein Bewertungsschema erarbeitet, das sich auf die kognitionspsychologischen Ansätze zur Schwierigkeit stützt. Durch einen Vergleich von theoretischer Schwierigkeit und durchschnittlicher Punktzahl des jeweiligen Lösungsansatzes, als Maß für dessen empirische Schwierigkeit, kann eine Aussage über die Validität einzelner Aspekte der erarbeiteten Methode zur Ermittlung der theoretischen Schwierigkeit getroffen werden. Eine Erweiterung der Methode auf komplette Modellierungsaufgaben, führt zur Beschreibung einer theoretischen Aufgabenschwierigkeit.

In der vorliegenden Arbeit werden fünf Modellierungsaufgaben entwickelt, von denen je drei zusammen in einem Testbooklet von circa 600 Gymnasialschülern der neunten Jahrgangsstufe bearbeitet werden. Für die Auswertung stehen circa 1800 Schülerlösungen zur Verfügung. Die Schülerlösungen werden in verschiedene Lösungsansätze eingeordnet und mit einem in der Arbeit entwickelten Bewertungsschemas bewertet. Die so ermittelte durchschnittliche Punktzahl eines Lösungsansatzes, wird mit der theoretischen Schwierigkeit des jeweiligen Lösungsansatzes, welche mit der in der Arbeit entwickelten Methode ermittelt wird, verglichen.

Die statistische Auswertung dieser Daten bestätigt die Vermutung, dass Denkoperationen, welche innerhalb eines Lösungsansatzes parallel durchgeführt werden müssen, zu einer Verkomplizierung führen. Werden Modellierungsaufgaben als Ganzes in den Blick genommen, zeigt sich auch hier eine Präferenz für einen solchen Zusammenhang zwischen parallelen Denkoperationen und Schwierigkeit. Allerdings deuten die Ergebnisse darauf hin, dass dieser weniger stark ausfällt. In diesem Zusammenhang unterstützen die Ergebnisse, das zumeist intuitive Vorgehen von Lehrkräften bei der Entwicklung eines Bewertungsschemas einer Mathematikaufgabe, bei dem wichtige Zwischenschritte einer Lösung identifiziert und bepunktet und anschließend zu einer Gesamtpunktzahl aufsummiert werden.

Die Ergebnisse, welche die Annahme einer Verkomplizierung durch Parallelität weitestgehend bestätigenden, stellen das Potential kognitionspsychologisch basierter Methoden im Bereich von Modellierungsaufgaben bezüglich Schwierigkeit 
und Bewertung heraus. Zusätzlich kann dadurch ein wesentlichen Beitrag geleistet werden, die komplexe Struktur von Schülerlösungen von Modellierungsaufgaben zu verstehen und zu nutzen. Vor allem aus Sicht einer Lehrkraft ist dies ein wichtiger Schritt hin zu einer besseren Handhabbarkeit von Modellierungsaufgaben und folglich, deren alltagsunterrichtlicher Integration. 



\section{Abstract}

Mathematical modelling can be seen, in simplified terms, as a process in which mathematics is used to elaborate on a reaslistic problem. Mathematical modelling is one of the six general mathematical competencies which are named in the German education standards for secondary education. At international level mathematical modelling is established in comparative studies, such as PISA (Programme for International Student Assessment). However, several studies confirm that the percentage of mathematical modelling in everyday school life is low. Regarding these findings, technical tasks are still the centre of mathematical teaching, wheareas modelling tasks seem to be of little importance. A detailed examination of the implications of curricular integration of mathematical modeling, reveals difficulties and obstacles which can be associated with special characteristics of modelling tasks. The variety of possible solutions for solving modelling tasks and the related applicability of different kinds of mathematical knowledge, means that, in many respects, modelling tasks are not easily manageable.

The disparity between educational requirements and everyday school teaching shows that especially in this area a need for action can be identified. The present thesis builds on this issue by working out difficulty-generating aspects based on actual student solutions, particularly from a cognitive psychological perspective. At first student solutions which are based e.g. on the same mathematical model, are clustered into solution approaches. The cognitive structure of solution approaches is then analysed for parallel and sequential thought operations. A hypothesis derived from cogntive psychology is that the number of parallel thought operations has an influence on the difficulty of the respective solution approach. The aim is to develop a valid method to determine the theoretical difficulty of solution approaches. In addition, an assessment scheme is developed which is based on the cognitive psychological approach used for the determination of difficulty. By comparison 
of theoretical difficulty and average score of the respective solution approach as a measure for the empirical difficulty, a statement can be made about the validity of different aspects of the developed method for the determination of difficulty. An extension of the method to complete modelling tasks results in a description of a theoretical task difficulty.

In the present thesis five modelling tasks have been developed. The test booklets consisted of three modelling tasks each and have been solved by ca. 600 high school stundets of the 9th grade (15 to 16 years of age). Thus, ca. 1800 student solutions were available for evaluation. The student solutions were classified into different solution approaches and scored on the basis of an assessment scheme developed within this thesis. The average score of a solution approach, as a measure for the empirical difficulty, has then been quantitatively compared to the respective theoretical difficulty which was determined by the developed method.

The statistical analysis of this data confirms the assumption that thought operations being proceessed in parallel within one solution approach, lead to a complication of the respective solution approach. In view of complete modelling tasks, the results show a similar preference for a relation between parallel thought operations and difficulty, however, this relation turns out to be less dominant. In this context, the results support a mostly intuitive approach of teachers in developing an assessment scheme of mathematics tasks by identifying and scoring important intermediate steps which are then added up to a total score.

The results which most closely confirm the hypothesis of a complication by parallelism of thought operationes, point out the potential of cognitive psychological based methods in the area of modelling tasks concerning both difficulty and assessment. Additionally a singificant contribution is made to understand and make use of the complex structure of student solutions of modelling tasks. Especially from a teachers' point of view this is an important step towards a better manageability and thus, an integration of modelling tasks to everyday school life. 


\section{Inhaltsverzeichnis}

1 Einleitung 1

I Theoretische Grundlagen 3

2 Mathematisches Modellieren $\quad 5$

2.1 Begriffsdefinition . . . . . . . . . . . . . 5

2.1.1 Mathematisches Modell . . . . . . . . . . . . 6

2.1.2 Mathematisches Modellieren . . . . . . . . . . 8

2.1.3 Modellierungskreislauf . . . . . . . . . . . . . . . 11

2.1 .4 Modellierungsaufgaben . . . . . . . . . . . . . 16

2.2 Zielsetzungen . . . . . . . . . . . . . . . . . . . . 19

3 Mathematisches Modellieren im Mathematikunterricht 25

3.1 Mathematisches Modellieren im Schulalltag . . . . . . . . . . 26

3.2 Schwierigkeiten und Hindernisse . . . . . . . . . . . . . . . . 27

3.2.1 Organisatorische Hindernisse . . . . . . . . . . . . 28

3.2.2 Schülerbezogene Hindernisse . . . . . . . . . . . . . . . . 29

3.2.3 Materialbezogene Hindernisse . . . . . . . . . . . . . 30

3.2.4 Lehrerbezogene Hindernisse . . . . . . . . . . . . . . . 31

3.3 Bewertung von Modellierungsaufgaben . . . . . . . . . . 33

3.4 Aufgabenschwierigkeit . . . . . . . . . . . . . 38

3.5 Folgerungen für die vorliegende Arbeit . . . . . . . . . . . 41 


\section{Forschungsansatz und Zielsetzung}

4 Forschungsfragen 45

5 Schwierigkeit von Modellierungsaufgaben 49

5.1 Die Struktur von Lösungsansätzen . . . . . . . . . . . . . . . 50

$5.1 .1 \quad$ Beziehung zu Modellbildungssoftware . . . . . . . . 53

5.1.2 Der Zusammenhang von Lösungsstruktur und Schwierigkeit 57

5.2 Denkstrukturanalyse . . . . . . . . . . . . . 58

5.2.1 Verortung von Denkoperationen in der Denkstruktur . . . 64

5.3 Denkstrukturen und das Arbeitsgedächtnis . . . . . . . . . . . . 70

5.4 Komplexität der Aufgabenstellung . . . . . . . . . . . . . . 72

5.5 Modelle zur Bestimmung der Schwierigkeit von Lösungsansätzen $\quad 77$

III Methode $\quad 85$

6 Konzeption der Studie $\quad 87$

6.1 Ablauf und Stichprobe . . . . . . . . . . . . . . . . . . 87

6.2 Aufgabenentwicklung . . . . . . . . . . . . . 89

6.2.1 Kriterien zur Entwicklung der Modellierungsaufgaben . . 90

6.3 Die Modellierungsaufgaben . . . . . . . . . . . . . . 91

$6.3 .1 \quad$ Taj Mahal _.................. 91



6.3 .3 Cola . . . . . . . . . . . . . . . . . 99 95

6.3 .4 Tennisschläger . . . . . . . . . . . . . . 97

6.3 .5 Brücke . . . . . . . . . . . . . . 99

7 Komplexität der Aufgabenstellung $\quad 103$

8 Kategorisierung von Schülerlösungen $\quad 107$

8.1 Lösungsansätze Taj Mahal _ . . . . . . . . . . . . . . . . . . . . 108

8.1 .1 Lösungsansatz Fläche . . . . . . . . . . . . . . . . . . 110

8.1.2 Lösungsansatz Zeilen/Spalten . . . . . . . . . . . 113

8.1 .3 Lösungsansatz Etage . . . . . . . . . . . . . . . . 116

9 Denkstrukturen und Schwierigkeitsgrad 119

9.1 Denkstrukturanalyse Taj Mahal . . . . . . . . . . . . . . . . 119

9.1 .1 Fläche . . . . . . . . . . . . . . . 121

9.1.2 Lösungsansatz Zeilen/Spalten . . . . . . . . . . . . . 124 
9.1.3 Lösungsansatz Etage . . . . . . . . . . . . . . . . . 126

9.2 Theoretische Schwierigkeit der Lösungsansätze Taj Mahal _ . . . 129

10 Bewertungsschema 131

10.1 Entwicklung eines Bewertungsschemas . . . . . . . . . . 132

10.2 Bewertungsschemata Taj Mahal . . . . . . . . . . . 133

10.2.1 Lösungsansatz Fläche . . . . . . . . . . . . . . . 134

10.2.2 Lösungsansatz Zeilen/Spalten . . . . . . . . . . . . . 137

10.2 .3 Etage . . . . . . . . . . . . . . . 139

IV Ergebnisse, Diskussion und Ausblick 141

11 Ergebnisse und Auswertung 143

11.1 Lösungsraum der Modellierungsaufgaben . . . . . . . . . . . 145

11.2 Empirische Schwierigkeit . . . . . . . . . . . . . . . . . . . . . 148

11.3 Vergleich von empirischer und theoretischer Schwierigkeit . . . 154

11.3.1 Regressionsmodelle und Gütemaß . . . . . . . . . . . . 154

11.3.2 Empirische und theoretische Schwierigkeit der Lösungsansätze . . . . . . . . . . . . . . 158

11.3.3 Empirische und theoretische Schwierigkeit der Modellierungsaufgaben . . . . . . . . . . . 167

12 Diskussion der Ergebnisse 173

12.1 Lösungsraum der Modellierungsaufgaben . . . . . . . . . . . 173

12.2 Analyse der empirischen Schwierigkeit . . . . . . . . . . . . 175

12.3 Analyse des theoretischen und empirischen Schwierigkeitsvergleichs auf Ebene der Lösungsansätze . . . . . . . . . . . . . . 176

12.4 Analyse des theoretischen und empirischen Schwierigkeitsvergleichs auf Ebene der Modellierungsaufgaben . . . . . . . . . 178

13 Zusammenfassung und Ausblick $\quad 181$

\section{Anhang}

$\begin{array}{ll}\text { A Test-Booklets } & 185\end{array}$

B Analyse der restlichen Modellierungsaufgaben 193

B.1 Modellierungsaufgabe Kartoffel . . . . . . . . . . . . . . . . . . . 194

B.1.1 Lösungsansätze . . . . . . . . . . . . . . . . . . . . . . . 194 
B.1.2 Denkstrukturanalyse . . . . . . . . . . . . . . . . 204

B.1.3 Bewertungsschemata . . . . . . . . . . . . 211

B.2 Modellierungsaufgabe Cola . . . . . . . . . . . . . . . . 216

B.2.1 Lösungsansätze . . . . . . . . . . . . . . . . 216

B.2.2 Denkstrukturanalyse . . . . . . . . . . . . . . . . 224

B.2.3 Bewertungsschemata . . . . . . . . . . . . . . . . 229

B.3 Modellierungsaufgabe Tennisschläger . . . . . . . . . . . 233

B.3.1 Lösungsansätze . . . . . . . . . . . . . . . . . 233

B.3.2 Denkstrukturanalyse . . . . . . . . . . . . . . . 241

B.3.3 Bewertungsschemata . . . . . . . . . . . . . . 246

B.4 Modellierungsaufgabe Brücke . . . . . . . . . . . . . 250

B.4.1 Lösungsansätze . . . . . . . . . . . . . . . . . . . . . 250

B.4.2 Denkstrukturanalyse . . . . . . . . . . . . 256

B.4.3 Bewertungsschemata . . . . . . . . . . . . . . 264

C Anhänge zur statistischen Auswertung 269

$\begin{array}{lr}\text { Abbildungsverzeichnis } & 279\end{array}$

$\begin{array}{ll}\text { Tabellenverzeichnis } & 285\end{array}$

$\begin{array}{ll}\text { Literaturverzeichnis } & 291\end{array}$ 


\section{Kapitel 1}

\section{Einleitung}

In der mathematikdidaktischen Diskussion findet das mathematische Modellieren seit einigen Jahrzehnten besondere Beachtung. Dies manifestiert sich nicht zuletzt insbesondere in der ersten und dritten von Winter (1995, S. 37) formulierten Grunderfahrung, welche insgesamt den Beitrag des Fachs Mathematik zur Allgemeinbildung herausstellen:

„(1) Erscheinungen der Welt um uns, die uns alle angehen oder angehen sollten, aus Natur, Gesellschaft und Kultur, in einer spezifischen Art wahrzunehmen und zu verstehen,

(2) mathematische Gegenstände und Sachverhalte, repräsentiert in Sprache, Symbolen, Bildern und Formeln, als geistige Schöpfungen, als eine deduktiv geordnete Welt eigener Art kennen zu lernen und zu begreifen,

(3) in der Auseinandersetzung mit Aufgaben Problemlösefähigkeiten, die über die Mathematik hinaus gehen, (heuristische Fähigkeiten) zu erwerben."

Als eine von sechs mathematischen Kompetenzen nimmt das mathematische Modellieren auch in den Bildungsstandards für das Fach Mathematik eine Schlüsselrolle ein (Kultusministerkonferenz, 2004). Auf internationaler Ebene wird dem mathematischen Modellieren als fester Bestandteil von Leistungsstudien wie PISA (Programme for International Student Assessment) oder TIMSS (Trends in International Mathematics and Science Study) eine besondere Bedeutung zuerkannt. 
Somit herrscht bildungspolitisch und fachdidaktisch weitestgehend Einigkeit über die Relevanz der unterrichtlichen Integration von mathematischem Modellieren. Verschiedene Studien deuten jedoch darauf hin, dass die unterrichtliche Wirklichkeit diesen Konsens nur bedingt widerspiegelt (siehe z.B. Drüke-Noe, 2014; Hiebert et al., 2003; Jordan et al., 2008; Kunter et al., 2006). Die Suche nach Gründen für diese Situation lässt ein breites Spektrum an Ansatzpunkten zu. So können u.a. organisatorische, material-, schüler- und lehrerbezogene Hindernisse identifiziert werden (Blum, 1996, S. 17ff), welche die unterrichtliche Integration von Modellierungsaufgaben erschweren. Aus Lehrerperspektive, als Exekutive bildungspolitischer und curricularer Vorgaben, haben mehrere Studien gezeigt, dass Aspekte wie Zeitumfang und Bewertung maßgebliche Hindernisse für die Integration von Modellierungsaufgaben darstellen (siehe z.B. Gainsbourg, 2008; Schmidt, 2009). Die Verwendungsmöglichkeit verschiedener Lösungsansätze führt dazu, dass Modellierungsaufgaben im Allgemeinen zunächst schwer zu überblicken sind. Dies kann dazu beitragen, dass deren unterrichtliche Integration als mit Schwierigkeiten behaftet angesehen wird. 